\title{
Heterochromatin base pair composition and diversification in holocentric chromosomes of kissing bugs (Hemiptera, Reduviidae)
}

\author{
Vanessa Bellini Bardella1, Sebastián Pita², André Luis Laforga Vanzela ${ }^{3}$, \\ Cleber Galvão ${ }^{4}$, Francisco Panzera²/+ \\ ${ }^{1}$ Universidade Estadual Paulista, Instituto de Biociências, Departamento de Biologia, Rio Claro, SP, Brasil \\ ${ }^{2}$ Universidad de la República, Facultad de Ciencias, Sección Genética Evolutiva, Montevideo, Uruguay \\ ${ }^{3}$ Universidade Estadual de Londrina, Centro de Ciências Biológicas, Departamento de Biologia Geral, Londrina, PR, Brasil \\ ${ }^{4}$ Instituto Oswaldo Cruz, Laboratório Nacional e Internacional de Referência em Taxonomia de Triatomíneos, Rio de Janeiro, RJ, Brasil

\begin{abstract}
The subfamily Triatominae (Hemiptera, Reduviidae) includes 150 species of blood-sucking insects, vectors of Chagas disease or American trypanosomiasis. Karyotypic information reveals a striking stability in the number of autosomes. However, this group shows substantial variability in genome size, the amount and distribution of C-heterochromatin, and the chromosome positions of $45 \mathrm{~S}$ rDNA clusters. Here, we analysed the karyotypes of 41 species from six different genera with C-fluorescence banding in order to evaluate the base-pair richness of heterochromatic regions. Our results show a high heterogeneity in the fluorescent staining of the heterochromatin in both autosomes and sex chromosomes, never reported before within an insect subfamily with holocentric chromosomes. This technique allows a clear discrimination of the heterochromatic regions classified as similar by C-banding, constituting a new chromosome marker with taxonomic and evolutionary significance. The diverse fluorescent patterns are likely due to the amplification of different repeated sequences, reflecting an unusual dynamic rearrangement in the genomes of this subfamily. Further, we discuss the evolution of these repeated sequences in both autosomes and sex chromosomes in species of Triatominae.
\end{abstract}

Key words: Heteroptera - Triatominae - holocentric chromosomes - CMA/DAPI banding - repetitive DNA

The subfamily Triatominae includes 150 species allocated to five tribes and 18 genera and belongs to the suborder Heteroptera (Galvão \& de Paula 2014). These insects are mostly distributed in the New World and are vectors of Chagas disease, which affects approximately six to seven million people worldwide, mostly in Latin America (WHO 2016). In addition to their medical importance, triatomines are a biological model in chromosome segregation analysis because they harbour holocentric chromosomes (with diffuse or non-localised centromeres) (Pérez et al. 2000). Unlike other hemipteran groups, members of the subfamily Triatominae present high uniformity in their diploid chromosome number. Almost all of the 90 studied species have 20 autosomes (A), with only three exceptions (Panzera et al. 2010). Chromosome variation is almost exclusively caused by the occurrence of different systems of sex chromosomes (XY, X1X2Y and X1X2X3Y). Although there is an extensive karyotypic stability for autosomal complement, the genome size as well as the amount and distribution of some repetitive DNAs, such as C-heterochromatin, and the chromosome positions of $45 \mathrm{~S}$ rDNA clusters are highly variable. These characteristics have

doi: 10.1590/0074-02760160044

Financial support: Udelar (CSIC - Project grant n 370 ), PEDECIBA and ANII (Uruguay). VB Bardella received funding from CAPES. + Corresponding author: fcopanzera@gmail.com

Received 10 February 2016

Accepted 19 July 2016 not been reported previously in other organisms with holocentric chromosomes (Panzera et al. 2010, 2012). The main source of karyotype differentiation in Triatominae is the constitutive heterochromatin variation revealed by the $\mathrm{C}$-banding technique. Constitutive heterochromatin is mainly composed of tandem (satellite DNA) and dispersed (transposable elements) repetitive DNA (Charlesworth et al. 1994). It is a key component in the structural and functional organisation of chromosomes. Heterochromatin is involved in various functions such as chromosome pairing, segregation, and even gene expression (Dimitri et al. 2009).

Heterochromatin variation in triatomines includes remarkable changes in the amount, size, chromosome location and behaviour of C-blocks during cell divisions (Panzera et al. 2010). The amount of heterochromatin varies over a wide range in the autosomal complement, from $45 \%$ (in Triatoma delpontei) to $0 \%$ in species of the matogrossensis and rubrovaria subcomplexes (Panzera et al. 2010). The size of heterochromatic blocks is also highly variable: they can be very tiny, as observed in Rhodnius pallescens (Gómez-Palacio et al. 2008), or they can constitute $80 \%$ of the entire chromosome, as in T. nitida (Panzera et al. 2010). Heterochromatic regions can be found in one, two or even in all autosomal pairs. Heterochromatic regions are generally located at one or both chromosomal ends, but in rare cases, interstitial bands are also observed (Panzera et al. 2014). Regarding sex chromosomes, all triatomine species show an almost entirely heterochromatic Y chromosome, while only a few species exhibit a C-heterochromatic X chromosome (Panzera et al. 2010, 2014). 
Considering the high diversity in the amount and distribution of C-heterochromatin in the autosomes and sex chromosomes of triatomines, there are few studies exploring the base composition of heterochromatic regions. To date, the heterochromatin base-pair richness has been studied in only four Triatoma species (Pérez et al. 2000, Bardella et al. 2010, 2014a). The aim of the present study was to compare the karyotypes of 41 species from six different genera according to the occurrence and distribution of DAPI/CMA bands, thereby allowing a better characterisation of heterochromatic regions in terms of their relative enrichment with $\mathrm{A}+\mathrm{T}$ or $\mathrm{G}+\mathrm{C}$ base pairs, respectively. With this strategy, we can suggest a broad overview of the evolution of these repeated sequences both in autosomes and in sex chromosomes in the subfamily Triatominae.

\section{MATERIALS AND METHODS}

Biological material - Table summarises the geographic origin, male diploid chromosome number and fluorescent banding results for the material analysed here. No specific permissions were required for the insect collections performed in this paper, and this study did not involve endangered or protected species. Chromosome preparations for C-CMA/DAPI banding were made from males and females of 41 triatomine species from the two principal tribes of the subfamily, Rhodniini and Triatomini, which include more than $90 \%$ of the 150 recognised species. For the Rhodniini tribe, which includes 13 species in two genera, we analysed two species: Rhodnius prolixus and $R$. pallescens. For Triatomini tribe we studied species from the following five genera: Dipetalogaster (one sp.), Eratyrus (one sp.), Mepraia (two spp.), Panstrongylus (five spp.) and Triatoma (30 spp.). By far, the most numerous genus is Triatoma, with 73 species. Within this genus, we studied the three main clades or groups: (a) the Rubrofasciata Group (from Central and North America and Old World species), (b) the Dispar Group (west of the Amazon Region), and (c) the Infestans Group (from south and east of the Amazon Region). Within each clade, we analysed various complexes and subcomplexes, according to the subdivisions proposed by Schofield and Galvão (2009) with modifications recently proposed by Pita et al. (2016).

Chromosome preparation and banding procedures Gonads (testes and ovaries) were fixed in ethanol-acetic acid (3:1) and softened in a $45 \%$ aqueous solution of acetic acid.

We applied a C-banding technique on squashed preparations according to Panzera et al. (2010). Then, the slides were treated with fluorochromes: $0.5 \mathrm{mg} / \mathrm{mL}$ chromomycin $\mathrm{A}_{3}$ (CMA) for $1.5 \mathrm{~h}$ and $2 \mu \mathrm{g} / \mathrm{mL}$ 4'-6-diamidino-2-phenylindole (DAPI) for $30 \mathrm{~min}$. Slides were mounted with a medium composed of glycerol/McIlvaine buffer, $\mathrm{pH} 7.0(1: 1, \mathrm{v}: \mathrm{v})$, plus $2.5 \mathrm{mM} \mathrm{MgCl}$, and preserved at $-20^{\circ} \mathrm{C}$. All slides were treated with both fluorochrome dyes (first CMA and then DAPI) in order to minimise the false-positive DAPI signals reported by Barros e Silva and Guerra (2010).

In all triatomine species, during meiotic prophase until diakinesis, the sex chromosomes always appear heteropycnotic with conventional staining and fluorescent banding. However, in metaphase plates, the sex chromosomes may have DAPI- or CMA-positive regions or similar fluorescent staining of the rest of the autosomes. The high fluorescent staining during the first meiotic prophase is consistent with the allocycly of these chromosomes during male meiosis, and it probably reflects a high degree of chromatin condensation rather than differences in base composition, although the latter has been suggested in other hemipteran species (Rebagliati et al. 2003). For this reason, we established the fluorescent patterns of sex chromosomes by analysing metaphases of both meiotic divisions and, when possible, in gonial mitosis. For autosomes, diplotene and early meiotic prophase were also analysed to detect small fluorescent regions that were not observed during metaphase due to high chromosome condensation. The pattern of fluorescence for each species was determined based on the chromosomal analyses of at least two individuals.

Microscopy and imaging - Slides were analysed under a Nikon Eclipse 80i epifluorescence microscope. Images were obtained with a Nikon DS-5 Mc-U2 digital cooled camera using Nikon Nis Elements 3.1 Advanced Research software and processed with Adobe Photoshop ${ }^{\circledR}$ software.

\section{RESULTS}

We observed a striking variation in fluorescent banding of the 41 analysed species. The table and figures show the detailed results by species. Considering the complexity and diversity of the observed fluorescent profiles, we present the results for the autosomes and for each sex chromosome (Xs and Y) separately.

Autosomal chromatin by fluorescent banding - In triatomines, all autosomal $\mathrm{C}$-heterochromatic regions always exhibited fluorescence staining, and we identified five fluorescence patterns.

Pattern 1 - Autosomal euchromatic regions without CMA/DAPI bands (Figs 1A-B, 2A-B, 3C-D, K-L, O-P). This is the most frequent pattern in triatomine species without autosomal C-heterochromatin, e.g., R. prolixus, T. boliviana, T. garciabesi, T. guasayana, T. klugi, T. longipennis, T. matogrossensis, T. rubrovaria, T. sordida Argentina, T. vandae, T. vitticeps and T. wygodzinsky) (Table).

Pattern 2 - Autosomal C-heterochromatic regions with co-localisation of $\mathrm{DAPI}^{+}$and $\mathrm{CMA}^{+}$fluorescence (Figs $1 \mathrm{~K}-\mathrm{L}, 2 \mathrm{O}-\mathrm{P}, 3 \mathrm{C}-\mathrm{H}$ ). This is the most frequent pattern observed among triatomine species with autosomal C-heterochromatin, being detected in several Panstrongylus species as well as in numerous species from the three main groups of Triatoma. The number and size of the fluorescent regions are variable in each triatomine species, and they are detailed in the table.

Pattern 3 - Autosomal C-heterochromatin regions exclusively $D A P I^{+}$. This pattern was observed in autosomes of $R$. pallescens, E. cuspidatus, T. bassolsae, T. ryckmani, T. maculata (Figs 1C-D, G-H, 2M-P) and some T. infestans (Andean lineage) and T. sordida La Paz (Fig. 3G-H).

Pattern 4 - Autosomal regions exclusively $\mathrm{CMA}^{+}$. Detected in Mepraia species (Fig. 1I-J), P. megistus (Fig. $1 \mathrm{M}-\mathrm{N}$ ) and brasiliensis subcomplex species (Fig. 3M-N). Furthermore, several species exhibited one autosomal pair with CMA dots (Figs 1E-F, 2K-L, 3I-J) (D. maxima, T. bas- 


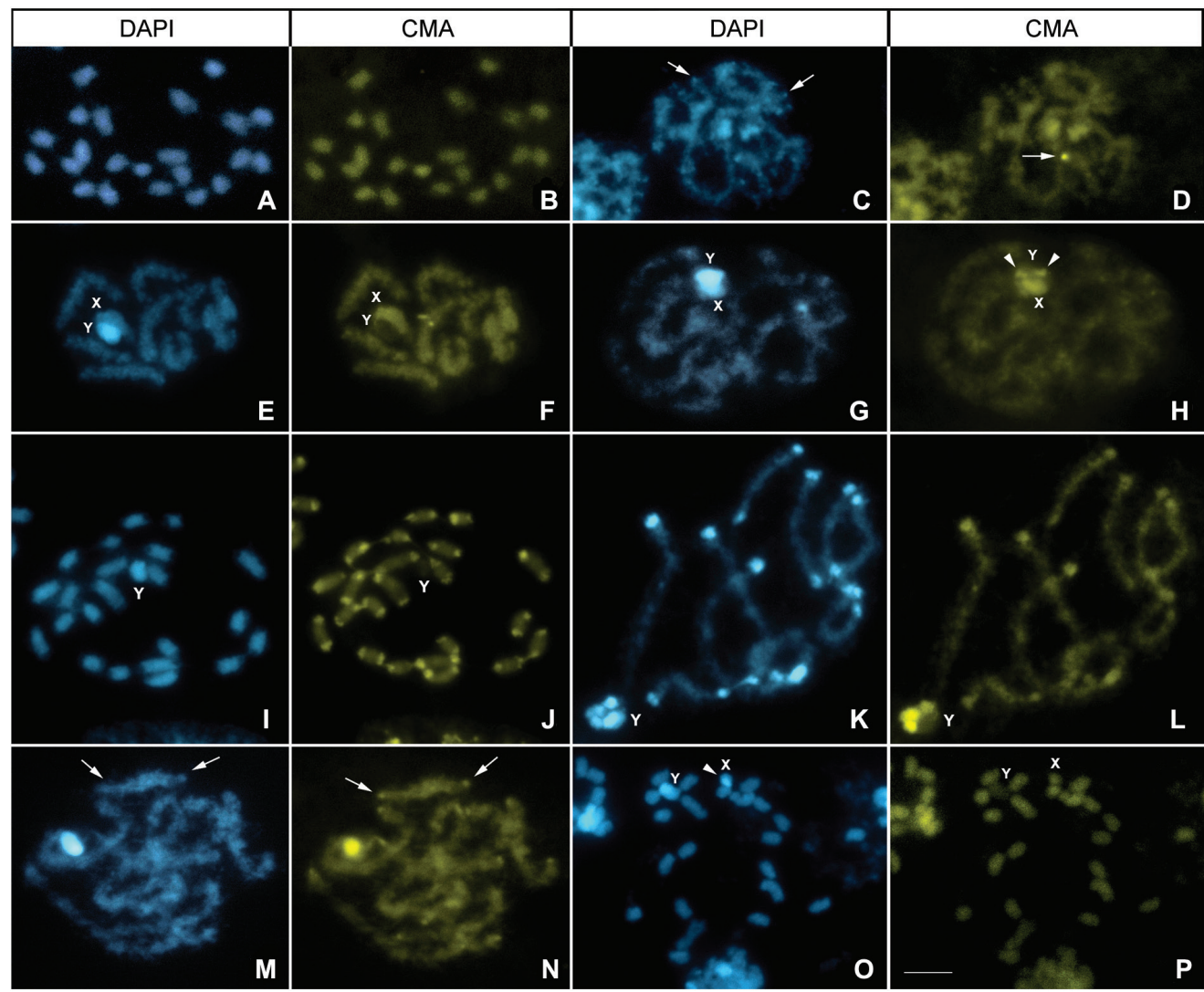

Fig. 1: fluorescent banding in several genera of Triatominae species, excepting Triatoma. (A-B) Rhodnius prolixus: spermatogonial prometaphase $(2 \mathrm{n}=22)$ without fluorescent signals in autosomes and sex chromosomes; (C-D) $R$. pallescens: diffuse stage showing DAPI and CMA autosomal dots (arrows); (E-F) Dipetalogaster maxima: pachytene with a CMA dot in one bivalent. The Y chromosome was completely CMA $/$ $\mathrm{DAPI}^{+}$, while the $\mathrm{X}$ chromosome did not exhibit fluorescent signals, similar to the case observed in other species; (G-H) Eratyrus cuspidatus: diffuse stage with a DAPI ${ }^{+}$dot in one bivalent. The Y chromosome was entirely DAPI ${ }^{+}$with a terminal CMA region (arrowhead); (I-J) Mepraia spinolai: spermatogonial prometaphase $(2 \mathrm{n}=23)$ showing 20 autosomes with CMA blocks in both chromosomal ends but no DAPI signal. The Y chromosome was CMA-/DAPI ${ }^{+}$, whereas both X chromosomes showed a terminal CMA band; (K-L) Panstrongylus chinai: early diplotene. Ten autosomal bivalents exhibited terminal co-localised $\mathrm{CMA}^{+} / \mathrm{DAPI}^{+}$regions in both chromosomal ends; (M-N) P. megistus: diffuse stage with $\mathrm{CMA}^{+}$dots in several bivalents (arrows); (O-P) P. geniculatus: spermatogonial prometaphase $(2 \mathrm{n}=23)$ without autosomal fluorescent regions. One X chromosome showed a DAPI ${ }^{+} \operatorname{dot}($ arrowhead). Bar: $10 \mu \mathrm{M}$.

solsae, T. costalimai, T. guazu and T. carcavalloi). In these species, except for D. maxima, the autosomal CMA regions are associated with the locations of ribosomal genes.

Pattern 5 - Autosomal C-heterochromatin region constituted by two adjacent sub-regions: a terminal $D A P I^{+}$and a subterminal $C M A^{+}$. This pattern was only observed in the three infestans subcomplex species (Fig. 3A-B). In brasiliensis subcomplex species, some autosomes presented an inverse pattern, i.e., terminal CMA regions with subterminal DAPI bands (Fig. 3M-N).

Despite the identification of these five autosomal fluorescence patterns, some species presented autosomes with more than one of the patterns described above. For example, T. sordida La Paz (Fig. 3G-H), species of the brasiliensis subcomplex (Fig. 3M-N) and T. infestans present at least two types of fluorescent profiles in their autosomes.

$X$ chromosome by fluorescent banding - Five fluorescent patterns were observed in the $\mathrm{X}$ chromosomes (Table). Most species showed euchromatic X chromo- somes without fluorescent bands (30 species), with unstained chromatin similar to that observed in the autosomal euchromatin. Undifferentiated $X$ chromosomes were observed in species with $\mathrm{XY}$ or $\mathrm{X}_{1} \mathrm{X}_{2} \mathrm{Y}$ sex systems among all genera studied (Figs 1-3). The other four patterns are associated with C-heterochromatin and involved the presence of different fluorescence bands, and they are as follows: (i) $\mathrm{CMA}^{+}$bands in both chromosomal ends of $\mathrm{X}_{1}$ and $\mathrm{X}_{2}$ chromosomes in $M$. spinolai (Fig. 1I-J); (ii) DAPI ${ }^{+}$bands in one chromosomal end of $\mathrm{X}_{1}$ and $\mathrm{X}_{2}$ chromosomes in T. ryckmani (Fig. 2M-N); (iii) $\mathrm{CMA}^{+}$with a $\mathrm{DAPI}^{+}$dot in a larger $\mathrm{X}$ chromosome of $T$. protracta and T. barberi (Fig. 2G$\mathrm{H}$ ); (iv) one $\mathrm{X}$ chromosome with a DAPI band in one end and a $\mathrm{CMA}^{+}$dot in the other chromosomal end $(P$. geniculatus and T. platensis) (Fig. 1O-P).

$Y$ chromosome by fluorescent banding - Two types of Y chromosomes were observed (Table). All Triatomini tribe species showed a Y chromosome that was entirely $\mathrm{DAPI}^{+}$(Figs 1E-P, 2, 3), while in Rhodniini species, the Y 


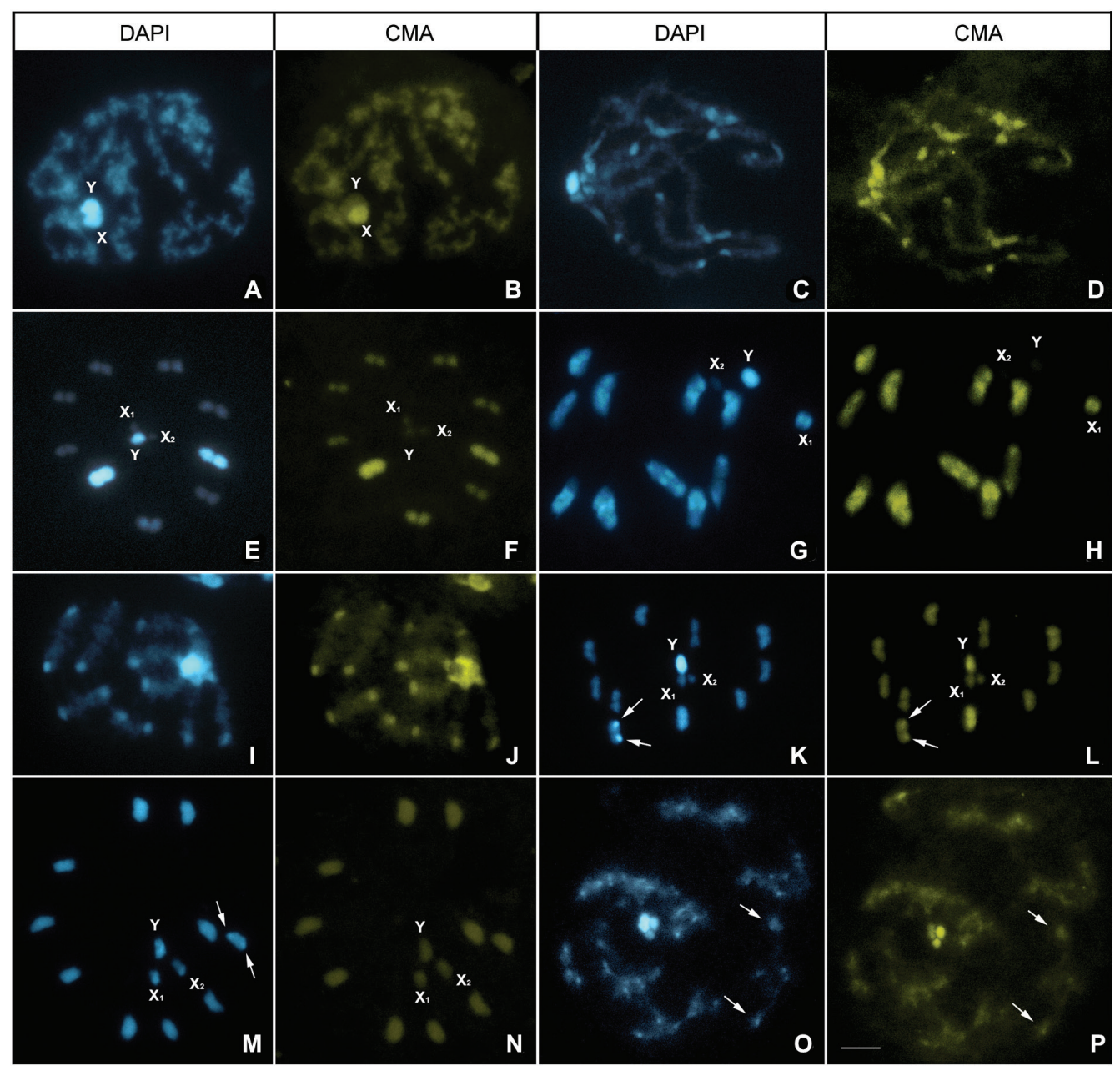

Fig. 2: fluorescent banding in several Triatoma species (Dispar and Rubrofasciata groups). (A-B) T. boliviana: diffuse stage without fluorescent signals in autosomes, and only the Y chromosome was completely DAPI stained; (C-D) T. rubrofasciata: diffuse stage showing all autosomes with $\mathrm{CMA}^{+} / \mathrm{DAPI}^{+}$regions co-localized; (E-F) T. nitida: metaphase II with two half-bivalents showing almost entirely co-localised CMA/DAPI regions. The Y chromosome was entirely DAPI', while both X chromosomes showed no fluorescent signals; (G-H) T. protracta: metaphase I with all bivalents showing terminal co-localised $\mathrm{CMA}^{+} / \mathrm{DAPI}^{+}$regions. The $\mathrm{Y}$ chromosome was $\mathrm{DAPI}^{+}$, while the larger $\mathrm{X}$ chromosome $\left(\mathrm{X}_{1}\right)$ showed only $\mathrm{CMA}^{+}$and $\mathrm{DAPI}^{+}$dots, and the $\mathrm{X}_{2}$ chromosome showed no fluorescence signals. (I-J) T. lecticularia: pachytene with all autosomes with co-localised $\mathrm{CMA}^{+} / \mathrm{DAPI}^{+}$regions; (K-L) T. bassolsae: metaphase II showing one half-bivalent with a co-localised $\mathrm{CMA}^{+} / \mathrm{DAPI}^{+}$dot (arrows); (M-N) T. ryckmani: metaphase II showing one half-bivalent with a DAPI ${ }^{+}$dot (arrows) and both $\mathrm{X}_{\text {chromosomes have terminal } \mathrm{DAPI}}{ }^{+}$ dots; (O-P) T. dimidiata: diplotene stage showing all bivalents with co-localised $\mathrm{CMA}^{+} / \mathrm{DAPI}^{+}$dots (arrows). Bar: $10 \mu \mathrm{M}$.

chromosome did not exhibit fluorescent signals (Fig. 1AD). In four species of the Triatomini tribe (E. cuspidatus, T. sordida Argentina, T. matogrossensis and T. vitticeps), the $\mathrm{DAPI}^{+} \mathrm{Y}$ chromosome also included a $\mathrm{CMA}^{+}$region.

\section{DISCUSSION}

The results presented here clearly show that the high chromosome variability displayed by C-banding in triatomines (for review see Panzera et al. 2010) is significantly increased with the application of fluorescent dyes. This technique allows a clear discrimination of the heterochromatic regions classified as similar by C-banding, thus constituting a new chromosome marker with taxonomic and evolutionary significance. An illustrative example is T. protracta and M. spinolai, two species exhibiting similar number, size and chromosome location of C-heterochromatic regions. However, the first species exhibited co-localised
AT-GC rich regions, while the second species showed GCrich bands exclusively (Figs 2G-H, 1I-J, respectively).

Reports on heterochromatin characterisation by DNA binding fluorochromes in Heteroptera are scarce and fragmentary, involving a small number of species from different families (Grozeva et al. 2004, Bressa et al. 2005, Chirino et al. 2013, Bardella et al. 2014b, c). Thus, its application as a taxonomic marker has not been successfully implemented. This technique has only recently been applied to differentiate cryptic species in the genus Macrolophus (Miridae) (Jauset et al. 2015).

This paper represents the most extensive C-fluorescence banding analyses applied to an insect group with holocentric chromosomes. We identified five autosomal fluorescent patterns in triatomines, some of them previously reported in other heteropteran species (Bressa et al. 2005, Bardella et al. 2014b, c, Bansal \& Kaur 2015). However, 


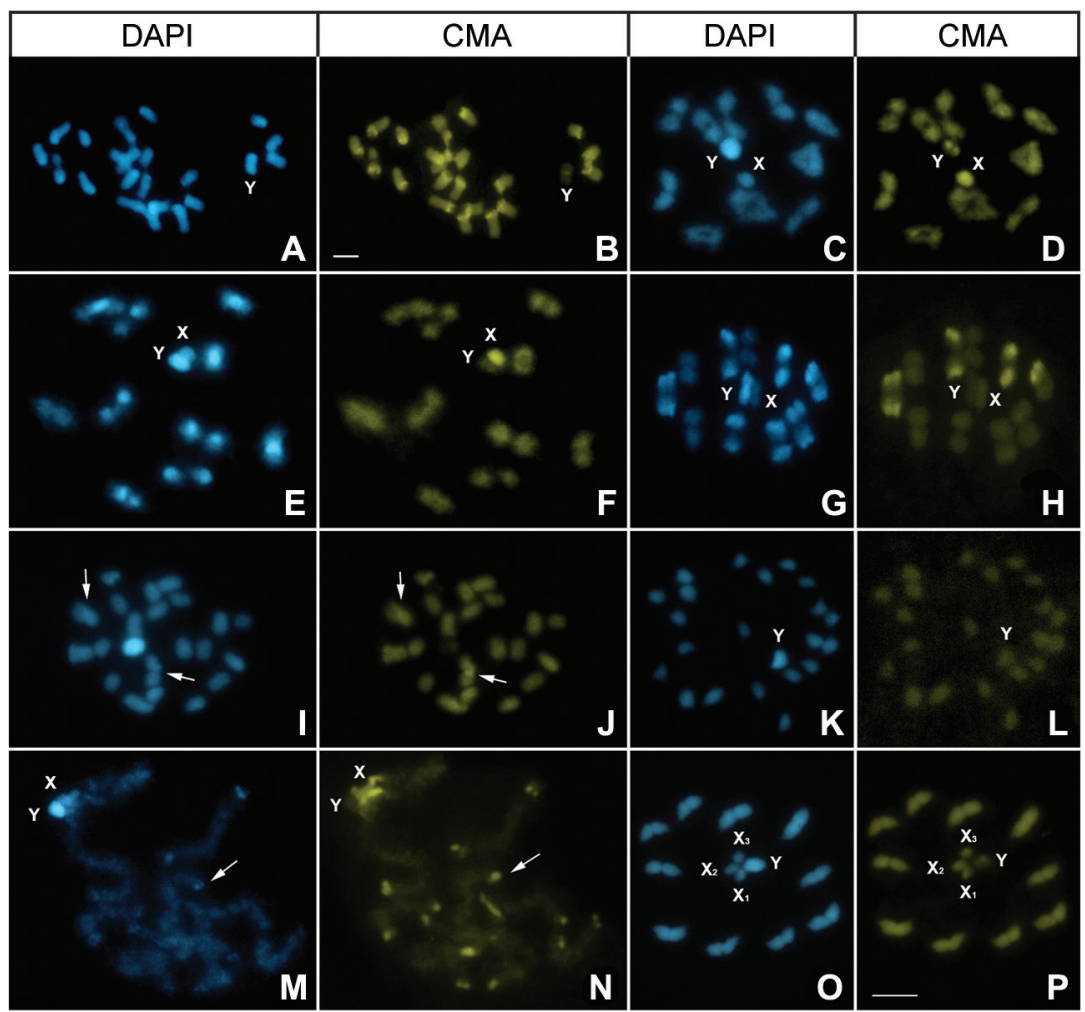

Fig. 3: fluorescent banding in several Triatoma species from South America (Infestans group). (A-B) T. delpontei: spermatogonial prometaphase $(2 \mathrm{n}=22)$ with all autosomes showing a large fluorescent block composed of two sub-regions: a terminal DAPI ${ }^{+}$region and a sub-terminal $\mathrm{CMA}^{+}$ region, in only one chromosomal end. (C-D) T. sordida Argentina: late diplotene showed no fluorescent bands on their ten autosomal pairs. The $\mathrm{Y}$ chromosome is DAPI ${ }^{+}$with $\mathrm{CMA}^{+}$dots, while the $\mathrm{X}$ chromosome is DAPI-negative but positive for CMA staining; (E-F) T. sordida sensu stricto: last diplotene. All bivalents showed $\mathrm{CMA}^{+} / \mathrm{DAPI}^{+}$regions co-localised. The $\mathrm{Y}$ chromosome was DAPI and negative for $\mathrm{CMA}^{+}$, while the $\mathrm{X}$ chromosome had the inverse staining pattern; (G-H) T. sordida La Paz: metaphase I with seven bivalents showed DAPI ${ }^{+}$blocks, four of them also with $\mathrm{CMA}^{+}$staining. The Y chromosome is entirely $\mathrm{DAPI}^{+}$, while the $\mathrm{X}$ chromosome had no fluorescent regions; (I-J) T. guazu: Spermatogonial prometaphase $(2 \mathrm{n}=22)$ without autosomal fluorescent bands, except one autosomal pair with an interstitial CMA $\mathrm{CM}^{+}$band (arrows); (K-L) $T$. wygodzinsky: spermatogonial prometaphase without autosomal fluorescent bands; (M-N) T. brasiliensis: pachytene stage. All autosomes showed terminal $\mathrm{CMA}^{+}$dots on both chromosomal ends. Some bivalents also presented sub-terminal $\mathrm{DAPI}^{+}$dots, adjacent to $\mathrm{CMA}^{+}$regions (arrows); (OP) T. vitticeps: metaphase II showing all autosomes without fluorescent bands. The Y chromosome was totally DAPI ${ }^{+}$with one terminal CMA ${ }^{+}$ dot. Furthermore, one X chromosome exhibited a DAPI dot, whereas the other two X chromosomes showed no fluorescent staining. Bar: $10 \mu \mathrm{M}$.

patterns four and five are uncommon in Heteroptera. Pattern four was recently described in the coreid Holhymenia histrio, but most of its regions are in intercalary position (Bardella et al. 2014b). Pattern five (and its inverse) has not been described in any other insect group (Fig. 3A-B, M-N).

Each fluorescent pattern reflects the presence of distinctive and specific sequences that compose the repetitive DNA. Furthermore, within the same pattern, different DNA repeats are likely to be involved, as observed in $T$. infestans (Bardella et al. 2014a). The diverse fluorescent patterns are probably due to the amplification of different repeated sequences, which reflects an extraordinary dynamic in the genomes of the Triatominae, never observed before in insects with holocentric chromosomes. This phenomenon contrasts with that observed in other heteropteran groups, in which each subfamily presents few heterochromatin types (Bressa et al. 2005, Bardella et al. 2014b).

Despite the striking variability in the fluorescent patterns, we observed that closely related species present similar compositions of A-T and/or C-G regions. For example, all species of the infestans subcomplex exhibited the same autosomal fluorescent profile (autosomal pattern five) (Fig. 3A-B, Table). Similar phenomena are observed among $\mathrm{Me}$ praia species, several Panstrongylus species, and in the brasiliensis, rubrovaria and protracta subcomplexes. At the evolutionary level, the occurrence of similar DNA repeats among closely related species suggests a common ancestor having a particular satellite DNA library. This feature is recurrent in related species and has been reported in different insect groups such as Coleoptera (Pons et al. 2004) and Orthoptera (Yoshimura et al. 2006). In triatomines, a similar process was suggested in infestans subcomplex species by GISH studies (Pita et al. 2014).

Diversity in X chromosome heterochromatin composition - Similar to other hemipteran groups, most triatomine species (30 out of 41 species) showed euchromatic $\mathrm{X}$ chromosomes without fluorescent bands. However, four fluorescent patterns on the $\mathrm{X}$ chromosomes were detected, involving species with $\mathrm{XY}, \mathrm{X}_{1} \mathrm{X}_{2} \mathrm{Y}$ and $\mathrm{X}_{1} \mathrm{X}_{2} \mathrm{X}_{3} \mathrm{Y}$ sex systems from three different genera (Figs 1-3). This paper reveals an extensive variability on the $X$ 


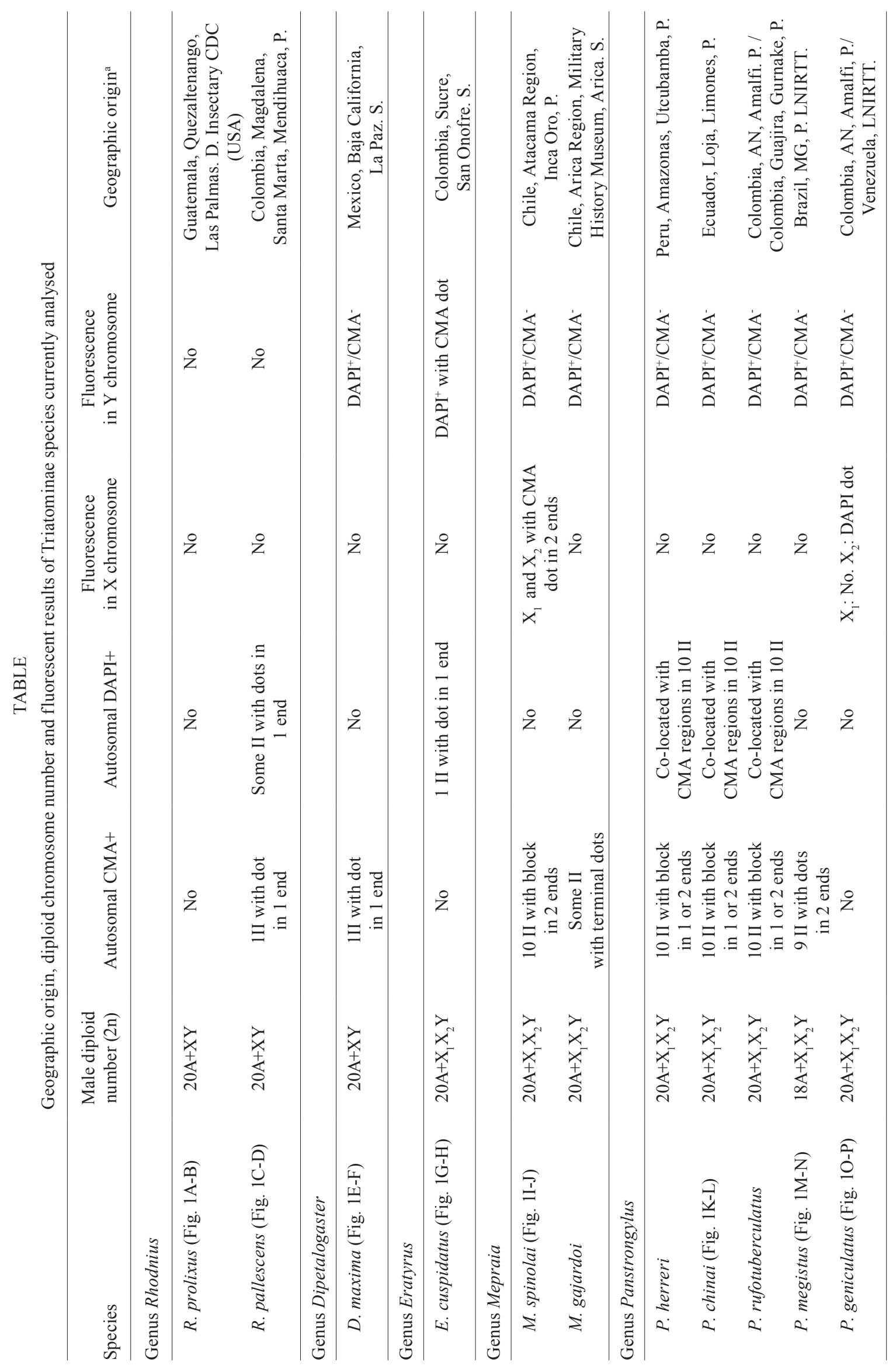




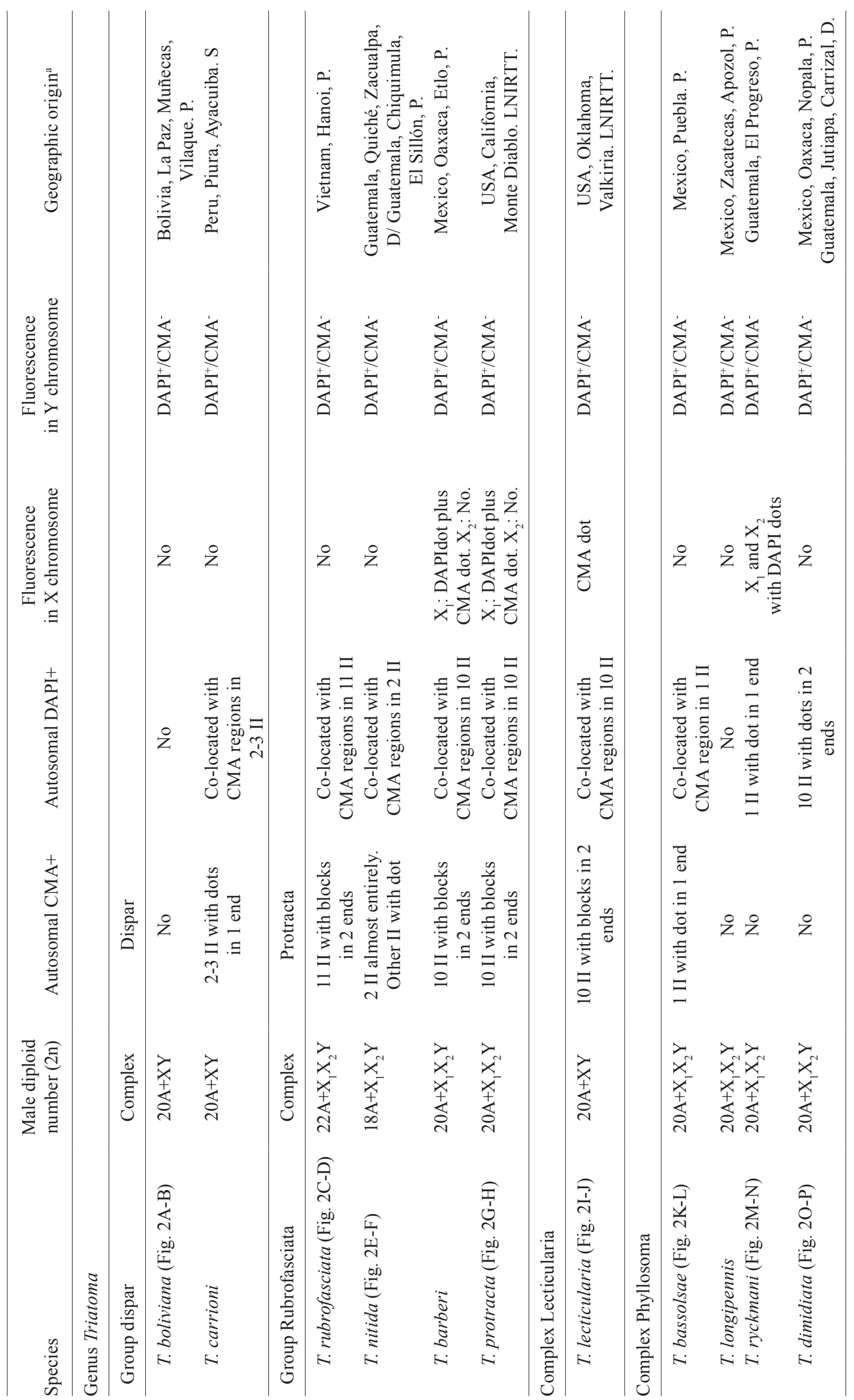




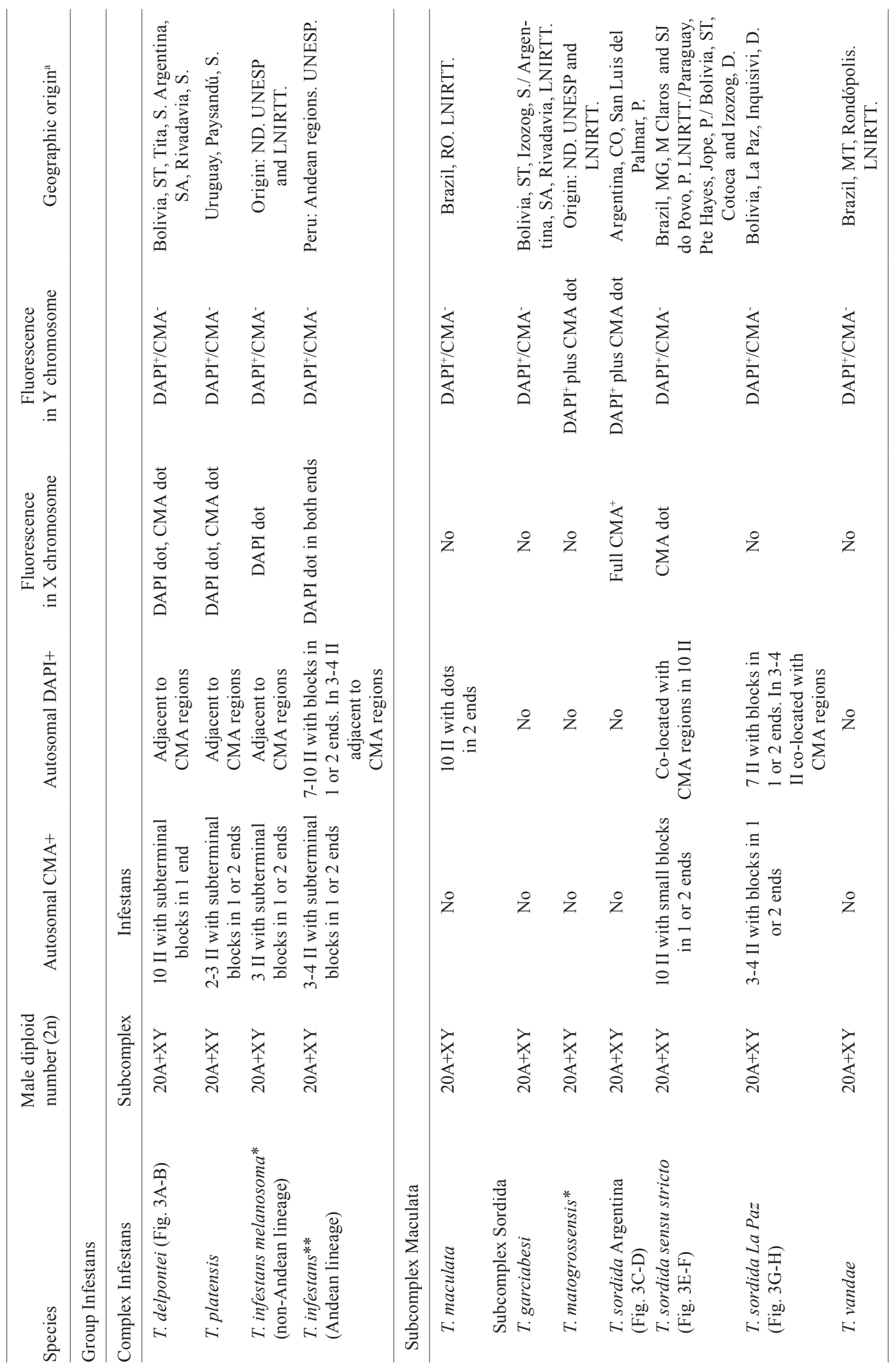




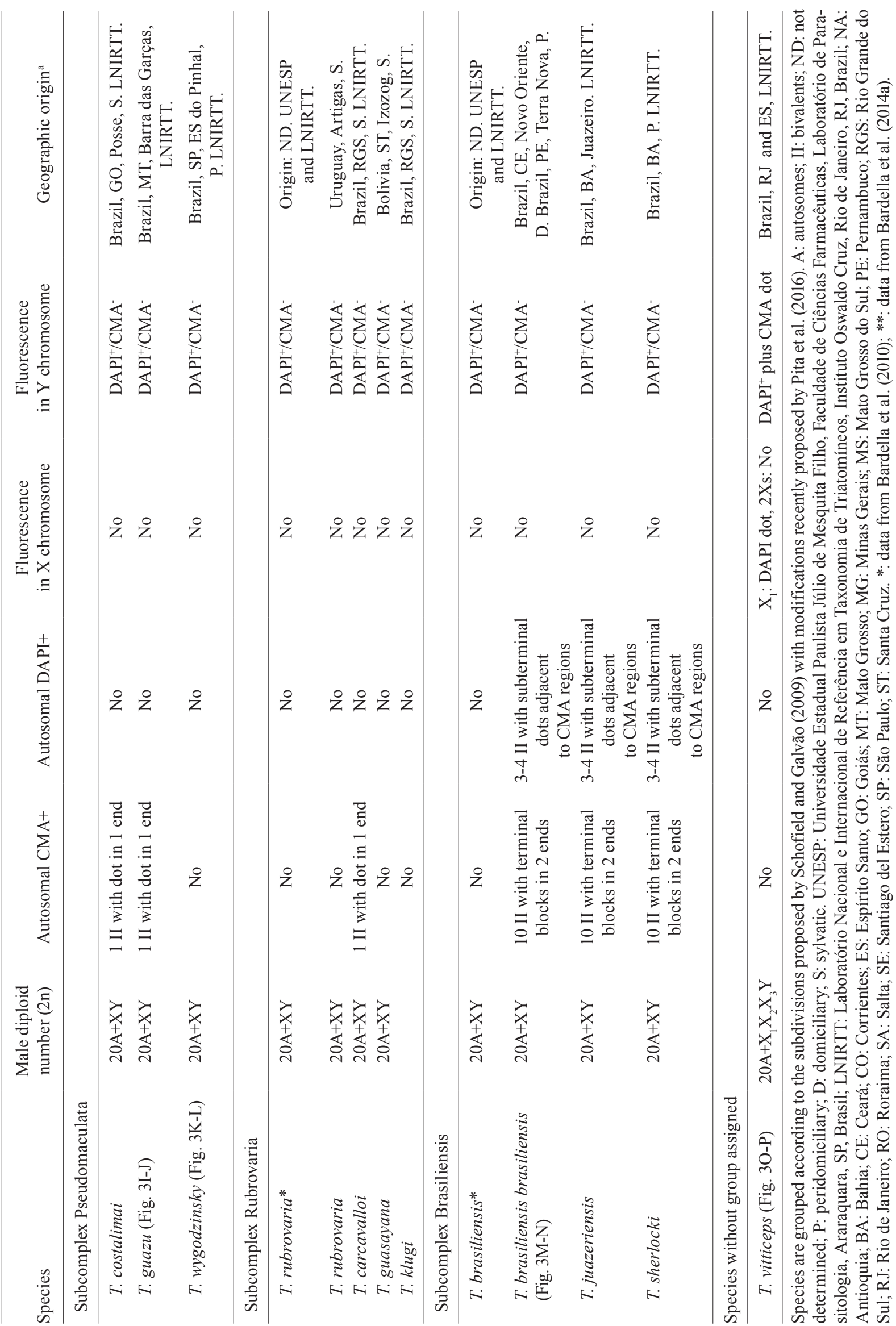


chromosomes not reported in other heteropteran groups. Only in the family Coreidae have some variations on the X chromosomes been described (Bardella et al. 2014b). Considering that the heterochromatic $\mathrm{X}$ chromosome is observed in evolutionarily distant triatomine species involving different sex mechanisms, its occurrence is probably due to convergent evolution. Therefore, the ancestral X chromosome should have been similar to the euchromatic autosomes without C-heterochromatic regions or fluorescent banding.

Variation in $Y$ chromosome heterochromatin composition - All Triatominae species currently analysed by conventional C-banding, approximately 90 species, showed a heterochromatic Y chromosome. However, fluorescent banding on Y chromosomes reveals a clear differentiation between the tribes Triatomini and Rhodniini. All species of Triatomini (39 species of five different genera, Table) showed an entirely $\mathrm{DAPI}^{+} \mathrm{Y}$ chromosome, revealing that this chromosome is composed of AT-rich repeated sequences (Figs 1E-P, 2, 3). This high similarity shared among the $\mathrm{Y}$ chromosomes of Triatomini species supports the idea that this chromosome, including the repeat sequences contained therein, is a tribe ancestral character (Pita et al. 2014). Only in four species that are not closely related (E. cuspidatus, $T$. sordida Argentina, T. matogrossensis and T. vitticeps) did the $\mathrm{DAPI}^{+}$ $\mathrm{Y}$ chromosome also include a $\mathrm{CMA}^{+}$region, which coincides with the existence of ribosomal genes (Bardella et al. 2010, Panzera et al. 2012, 2015). Considering the low frequency of species with $\mathrm{Y}$ chromosomes carrying $\mathrm{CMA}^{+}$regions and the presence of $\mathrm{CMA}^{+}$regions in evolutionarily distant species, we can conclude that this trait is a derivative character, at least for the Triatomini tribe. In addition, as $\mathrm{CMA}^{+} \mathrm{Y}$ chromosomes are correlated with the localisation of ribosomal genes, the same hypothesis may apply. In contrast, the Y chromosome in the Rhodniini tribe does not exhibit DAPI staining, probably indicating that this chromosome is composed of other repeated sequences (Fig. 1A-D). Although $R$. pallescens has ribosomal genes on both sex chromosomes (Pita et al. 2013), we do not observe $\mathrm{CMA}^{+}$bands on them.

In summary, the similar fluorochrome composition of the Y chromosome in Triatomini tribe and their striking differentiation with Rhodniini species detected here are consistent with GISH results (Pita et al. 2014). This chromosomal change is likely a consequence of evolutionary differentiation between these two tribes and may be useful as a phylogenetic marker.

Contradictory hypotheses support Triatominae as a monophyletic (Hypša et al. 2002, Weirauch \& Munro 2009, Patterson \& Gaunt 2010), polyphyletic (Schofield \& Galvão 2009) or paraphyletic group (Hwang \& Weirauch 2012). According to the latter models, the Triatomini and Rhodniini tribes are derived from quite different reduviid subfamilies. Considering that the split between the Rhodniini and Triatomini tribes is the most ancient known divergence point within triatomine bugs (between 40 and 95 million years ago) (Justi et al. 2016, Patterson \& Gaunt 2010, respectively), comparative molecular analyses of the Y chromosome among triatomines with puta- tive/suspected sister reduviid species may help to clarify the monophyletic or polyphyletic origin of Triatominae subfamily. For example, Zelurus femoralis, belonging to a Reduviinae genus considered to be a monophyletic group with Triatominae and Stenopodainae (Weirauch \& Munro 2009), presented a DAPI ${ }^{+} \mathrm{Y}$ chromosome similar to that of the Triatomini tribe (Poggio et al. 2013).

\section{REFERENCES}

Bansal N, Kaur H. C-heterochromatin distribution and its base composition in four species of Mictini (Heteroptera, Coreidae, Coreinae). Cytologia. 2015; 80(4): 405-13.

Bardella VB, Gaeta ML, Vanzela ALL, Azeredo-Oliveira MTV. Chromosomal location of heterochromatin and 45S rDNA sites in four South American triatomines (Heteroptera: Reduviidae). Comp Cytogenet. 2010; 4(2): 141-9.

Bardella VB, Grazia J, Fernandes JAM, Vanzela ALL. High diversity in CMA/DAPI banding patterns in heteropterans. Cytogenet Genome Res. 2014b; 142(1): 46-3.

Bardella VB, Rosa JA, Vanzela ALL. Origin and distribution of ATrich repetitive DNA families in Triatoma infestans (Heteroptera). Infect Genet Evol. 2014a; 23: 106-14.

Bardella VB, Sampaio TR, Venturelli NB, Dias AL, Giuliano-Caetano L, Fernandes JAM, et al. Physical mapping of $18 \mathrm{~S}$ rDNA and heterochromatin in species of family Lygaeidae (Hemiptera: Heteroptera). Genet Mol Res. 2014c; 13(1): 2186-99.

Barros e Silva AE, Guerra M. The meaning of DAPI bands observed after C-banding and FISH procedures. Biotech \& Histochem. 2010; 85(2): 115-25.

Bressa MJ, Larramendy ML, Papeschi AG. Heterochromatin characterization in five species of Heteroptera. Genetica. 2005; 124(2-3): 307-17.

Charlesworth B, Sniegowski P, Stephan W. The evolutionary dynamics of repetitive DNA in eukaryotes. Nature. 1994; 371(6494): 215-20.

Chirino MG, Papeschi AG, Bressa MJ. The significance of cytogenetics for the study of karyotype evolution and taxonomy of water bugs (Heteroptera, Belostomatidae) native to Argentina. Comp Cytogenet. 2013; 7(2): 111-29.

Dimitri P, Caizzi R, Giordano E, Accardo MC, Lattanzi G, Biamonti G. Constitutive heterochromatin: a surprising variety of expressed sequences. Chromosoma. 2009; 118(4): 419-35.

Galvão C, de Paula AS. Sistemática e evolução dos vetores. In: Galvão C, org. Vetores da doença de Chagas no Brasil [Internet]. Curitiba: Sociedade Brasileira de Zoologia; 2014. p. 2632. Available from: http://books.scielo.org/id/mw58j/pdf/galvao-9788598203096-03.pdf.

Gómez-Palacio A, Jaramillo-Ocampo N, Triana-Chávez O, Saldaña A, Calzada J, Pérez R, et al. Chromosome variability in the Chagas disease vector Rhodnius pallescens (Hemiptera, Reduviidae, Rhodniini). Mem Inst Oswaldo Cruz. 2008; 103(2): 160-4.

Grozeva S, Kuznetsova VG, Nokkala S. Patterns of chromosome banding in four nabid species (Heteroptera, Cimicomorpha, Nabidae) with high chromosome number karyotypes. Hereditas. 2004; 140(2): 99-104.

Hwang WS, Weirauch C. Evolutionary history of assassin bugs (Insecta: Hemiptera: Reduviidae): insights from divergence dating and ancestral state reconstruction. PLoS ONE. 2012; 7(9): e45523.

Hypša V, Tietz DF, Zrzavy J, Rego ROM, Galvão C, Jurberg J. Phylogeny and biogeography of Triatominae (Hemiptera: Reduviidae): molecular evidence of a New World origin of the Asiatic clade. Mol Phylogen Evol. 2002; 23(3): 447-57. 
Jauset AM, Edo-Tena E, Castane C, Agusti N, Alomar O, Grozeva S. Comparative cytogenetic study of three Macrolophus species (Heteroptera, Miridae). Comp Cytogenet. 2015; 9(4): 613-23.

Justi SA, Galvão C, Schrago CG. Geological changes of the Americas and their influence on the diversification of the Neotropical kissing bugs (Hemiptera: Reduviidae: Triatominae). PLoS Negl Trop Dis. 2016; 10(4): e0004527.

Panzera F, Ferreiro MJ, Pita S, Calleros L, Pérez R, Basmadjián Y, et al. Evolutionary and dispersal history of Triatoma infestans, main vector of Chagas disease, by chromosomal markers. Infect Genet Evol. 2014; 27: 105-13.

Panzera F, Pérez R, Panzera Y, Ferrandis I, Ferreiro MJ, Calleros L. Cytogenetics and genome evolution in the subfamily Triatominae (Hemiptera, Reduviidae). Cytogenet Genome Res. 2010; 128(1-3): 77-87.

Panzera F, Pita S, Nattero J, Panzera Y, Galvão C, Chavez T, et al. Cryptic speciation in the Triatoma sordida subcomplex (Hemiptera, Reduviidae) revealed by chromosomal markers. Parasit Vectors. 2015; 8: 495.

Panzera Y, Pita S, Ferreiro MJ, Ferrandis I, Lages C, Pérez R, et al. High dynamics of rDNA cluster location in kissing bug holocentric chromosomes (Triatominae, Heteroptera). Cytogenet Genome Res. 2012; 138(1): 56-67.

Patterson JS, Gaunt MW. Phylogenetic multi-locus codon models and molecular clocks reveal the monophyly of haematophagous reduviid bugs and their evolution at the formation of South America. Mol Phylogen Evol. 2010; 56(2): 608-21.

Pérez R, Rufas J, Suja J, Panzera F. Meiosis in holocentric chromosomes: orientation and segregation of an autosome and sex chromosomes in Triatoma infestans (Heteroptera). Chromosome Res. 2000; 8(1): 17-25.

Pita S, Lorite P, Nattero J, Galvão C, Alevi KCC, Teves SC, et al. New arrangements on several species subcomplexes of Triatoma genus based on the chromosomal position of ribosomal genes (Hemiptera - Triatominae). Infect Genet Evol. 2016; 43: 225-31.
Pita S, Panzera F, Ferrandis I, Galvão C, Gómez-Palacio A, Panzera Y. Chromosomal divergence and evolutionary inferences in Rhodniini based on the chromosomal location of ribosomal genes. Mem Inst Oswaldo Cruz. 2013; 108(3): 376-82.

Pita S, Panzera F, Sánchez A, Panzera Y, Palomeque T, Lorite P. Distribution and evolution of repeated sequences in genomes of Triatominae (Hemiptera-Reduviidae) inferred from genomic in situ hybridization. PLoS ONE. 2014; 9(12): e114298.

Poggio MG, Provecho YM, Papeschi AG, Bressa MJ. Possible origin of polymorphism for chromosome number in the assassin bug Zelurus femoralis longispinis (Reduviidae: Reduviinae). Biol J Linn Soc. 2013; 110(4): 757-64.

Pons J, Bruvo B, Petitpierre E, Plohl M, Ugarkovic D, Juan C. Complex structural features of satellite DNA sequences in the genus Pimelia (Coleoptera: Tenebrionidae): random differential amplification from a common 'satellite DNA library'. Heredity. 2004; 92(5): 418-27.

Rebagliati PJ, Papeschi AG, Mola LM. Meiosis and fluorescent banding in Edessa meditabunda and E. rufomarginata (Heteroptera: Pentatomidae: Edessinae). Eur J Entomol. 2003; 100(1): 11-8.

Schofield CJ, Galvão C. Classification, evolution, and species groups within the Triatominae. Acta Trop. 2009; 110(2-3): 88-100.

Weirauch C, Munro JB. Molecular phylogeny of the assassin bugs (Hemiptera: Reduviidae), based on mitochondrial and nuclear ribosomal genes. Mol Phylogen Evol. 2009; 53(1): 287-99.

WHO - World Health Organization. Chagas disease (American trypanosomiasis) [Internet]. 2016. Available from: http://www.who. int/mediacentre/factsheets/fs340/en/index.html.

Yoshimura A, Nakata A, Mito T, Noji S. The characteristics of karyotype and telomeric satellite DNA sequences in the cricket, Gryllus bimaculatus (Orthoptera, Gryllidae). Cytogenet Genome Res. 2006; 112(3-4): 329-6. 